\title{
Regional Cerebral Blood Flow in the Posterior Cingulate and Precuneus and the Entorhinal Cortical Atrophy Score Differentiate Mild Cognitive Impairment and Dementia Due to Alzheimer Disease
}

\author{
(D) B. Thomas, (D) R. Sheelakumari, (D) S. Kannath, (D) S. Sarma, and (D) R.N. Menon
}

\begin{abstract}
BACKGROUND AND PURPOSE: Alzheimer disease is the most common degenerative dementia affecting humans and mild cognitive impairment is considered the forerunner of this devastating illness with variable progression. Differentiating between them has become all the more essential with the advent of disease-modifying medications. The aim of this study was to test the utility of the entorhinal cortical atrophy score in combination with quantitative CBF in the posterior cingulate and precuneus using arterial spin-labeling to differentiate mild cognitive impairment and early Alzheimer disease.
\end{abstract}

MATERIALS AND METHODS: We analyzed MR imaging from a prospective data base of 3 age-matched groups: 21 cognitively healthy controls, 20 patients with mild cognitive impairment, and 19 patients with early Alzheimer disease. The highest entorhinal cortical atrophy score and an atlas-based measurement of CBF in the posterior cingulate and precuneus were estimated in these groups. Statistical comparison was performed among the groups for disease-prediction probability with these parameters independently and in combination using a binary logistic regression model.

RESULTS: The entorhinal cortical atrophy score performed well in distinguishing AD from HC, with a predicted probability of .887 (area under the curve, $P<.001$ ). The mean CBF of the posterior cingulate and precuneus was also found to be a useful discriminator (area under the curve, $0.810, P=<.001$ ). Combining the entorhinal cortical atrophy score and CBF was the best predictor (area under the curve, 0.957, $P<.001$ ). In distinguishing mild cognitive impairment and Alzheimer disease, entorhinal cortical atrophy also did well with an area under the curve of $0.838(P<.001)$. However regional CBF was not useful in differentiating them (area under the curve $=0.589, P=.339$ ). Entorhinal cortical atrophy scored poorly in distinguishing mild cognitive impairment from healthy controls (AUC $=0.571, P=.493)$, but CBF fared well, with an area under the curve of $0.776(P=.002)$.

CONCLUSIONS: Combining entorhinal cortical atrophy and regional CBF could be a potential imaging biomarker in distinguishing mild cognitive impairment and Alzheimer disease.

ABBREVIATIONS: ACE-M = South Indian language of Malayalam version of Addenbrooke's Cognitive Examination; $A D=$ Alzheimer disease; $A S L=$ arterial spin-labeling; ERICA = entorhinal cortical atrophy; $\mathrm{HC}=$ healthy controls; $\mathrm{MCI}=$ mild cognitive impairment; $\mathrm{PCG}=$ posterior cingulate gyrus; $\mathrm{PC}=$ precuneus; $\mathrm{ROI}=$ region of interest

Received April 22, 2019; accepted after revision August 1.

From the Department of Imaging Sciences and Interventional Radiology (B.T., R.S., S.K.), Division of Cognitive and Behavioural Neurology (R.N.M.), and Achutha Menon Centre for Health Sciences Studies (S.S.), Sree Chitra Tirunal Institute for Medical Sciences and Technology, Thiruvananthapuram, Kerala, India.

This work was supported by CSI/90/2012, SATYAM/278/2015, and SERB N-PDF 2017 grants from the Government of India.

Please address correspondence to Bejoy Thomas, MD, DNB, PDCC, Department of Imaging Sciences and Interventional Radiology, Sree Chitra Tirunal Institute for Medical Sciences and Technology, Thiruvananthapuram, Kerala, India; e-mail: bejoy@sctimst.ac.in; @drbejoy2002

-- Indicates open access to non-subscribers at www.ajnr.org http://dx.doi.org/10.3174/ajnr.A6219 burden for care of the affected. ${ }^{1,2}$ Mild cognitive impairment (MCI) is considered the forerunner of this devastating illness with variable progression. ${ }^{3}$ With the advent of disease-modifying medications, early detection and classification of preclinical dementia have become essential. Exploration of possible biomarkers identifying individuals who are at high risk for developing $\mathrm{AD}$ is the focus of current research. Validated biomarkers for diagnosing MCI at risk of progression include Pittsburgh compoundbased PET and CSF biomarkers, which are either not available in many centers worldwide or are prohibitively expensive. ${ }^{4}$ The relevance of validating instruments from widely available multimodality imaging techniques has been demonstrated by our group previously. ${ }^{5}$ 


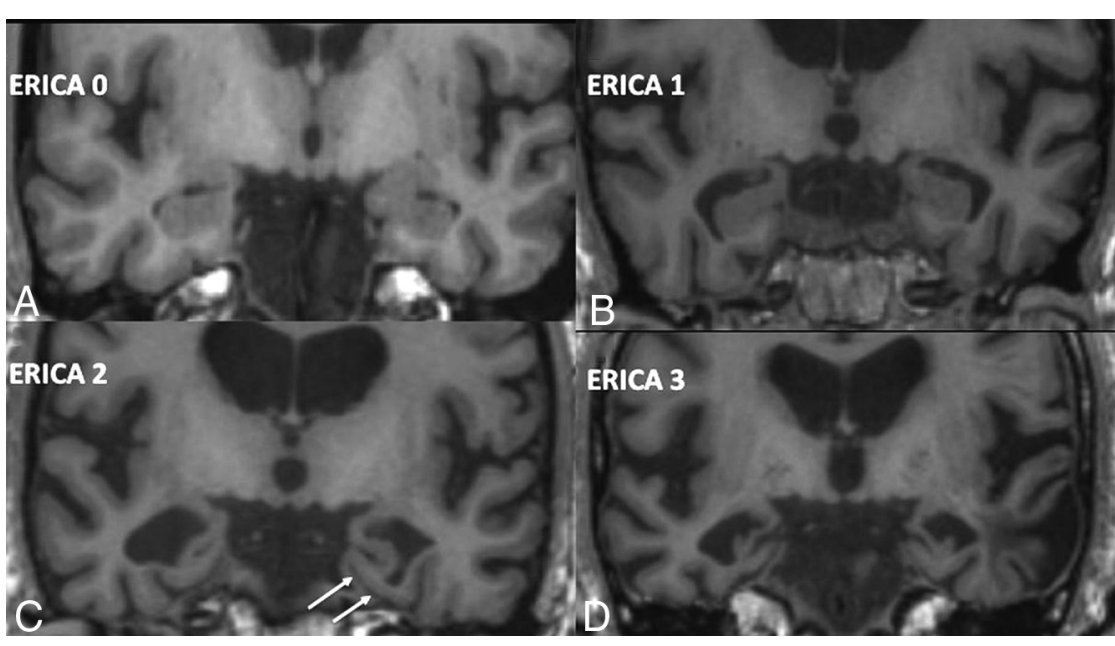

FIG 1. ERICA scoring. A, Score 0 with no parahippocampal atrophy. $B$, Score 1 with mild atrophy with prominence of collateral sulcus. C, Score 2 with moderate entorhinal cortical atrophy with tentorial cleft sign (arrows). D, Score 3 with marked parahippocampal atrophy. Adapted from Enkirch et al. ${ }^{17}$

Cerebral blood flow alterations in patients with $\mathrm{AD}$ and MCI have been identified as an important marker in understanding the neurophysiologic changes even before development of neuronal loss. ${ }^{6}$ Arterial spin-labeling (ASL) uses tagged arterial blood as an endogenous contrast for detecting regional CBF perfusion changes. Many recent studies have highlighted its role in imaging $\mathrm{AD}$ and $\mathrm{MCI}$, in which different brain regions have been found to show hypo- or hyperperfusion. ${ }^{7-13}$ ASL is a noninvasive MR perfusion technique, without the use of any ionizing radiation, intravenous contrast agents, or radioactive isotopes, and it is considered a potential alternative to FDG-PET imaging. ${ }^{14,15}$ Using ASL, many studies have consistently shown statistically significant hypoperfusion involving the posterior cingulate gyrus (PCG) and precuneus (PC), among many regions studied in MCI-AD. ${ }^{10,11,13}$ Only a few studies have correlated quantitative $\mathrm{CBF}$ with neuropsychology in $\mathrm{AD}$ and $\mathrm{MCI} .{ }^{16}$ No consistent correlation has been established between $\mathrm{CBF}$ and gray matter volume changes in the corresponding regions in patients with $\mathrm{AD}$ and $\mathrm{MCI}$ in the existing literature. ${ }^{13}$

The entorhinal cortex has been shown to undergo graded atrophy in the MCI-AD complex and is thought to be one of the early areas of the brain that shows gray matter atrophy in $\mathrm{AD}^{17} \mathrm{~A}$ recently described numeric atrophy scale of the entorhinal cortex (ERICA) score has been shown to correlate well with the cognitive changes and the diagnosis of $\mathrm{AD}^{17}$ It has also been shown to be better than the previously described medial temporal lobe atrophy score in differentiating $\mathrm{AD}$ from subjectively reported cognitive impairment in patients. ${ }^{17}$ The ERICA score is a 4-point atrophy rating scale from 0 to 3 , where 0 indicates no evidence for atrophy of the entorhinal cortex, and 3, marked atrophy (Fig 1). This was shown to be handy because the scoring system is very simple, but powerful enough to be used for the distinction of these conditions. However, this scoring system has not yet been validated by further studies.
The aim of the present study was to test the hypothesis that the regional $\mathrm{CBF}$ changes measured in the posterior cingulate and precuneus (PCG + $\mathrm{PC})$ region using the $3 \mathrm{D}$ fast spinecho pseudocontinuous ASL sequence along with ERICA scoring of entorhinal cortical atrophy will be a better biomarker in differentiating patients with $\mathrm{AD}$ and $\mathrm{MCI}$ compared with healthy controls (HC).

\section{MATERIALS AND METHODS}

MR images from the data base of prospectively recruited consecutive subjects attending the Memory and Neurobehavioral Disorders Clinic of a tertiary referral teaching hospital in the South Indian state of Kerala, between 2016 and 2018, were included in this study. Age-matched control data were also collected during the period from the data base. Five subjects were excluded due to insufficient image quality. The final sample consisted of 21 controls, 20 patients with MCI, and 19 patients with AD. All subjects provided written consent according to procedures approved by the Institutional Ethics Committee of our institution. The diagnosis of $\mathrm{MCI}$ and $\mathrm{AD}$ was based on subjective cognitive symptoms verified by objective evidence of decline by a family member compared with the previous status and confirmed by evidence of a decline in $\geq 1$ neuropsychological domain ${ }^{4}$ as shown below. Participants with a global cognitive assessment score, as determined on the vernacular version (in the South Indian language of Malayalam of Addenbrooke's Cognitive Examination [ACE-M]), between 88 and 100; a Clinical Dementia Rating score of 0 ; with formal education of $>10$ years; no subjective memory symptoms; and with no serious neurologic/psychiatric issues were selected as healthy controls. ${ }^{18,19}$ Participants with a Clinical Dementia Rating of $<2$ and an ACE$\mathrm{M}$ score between 60 and 78 were confirmed as having $\mathrm{AD}$ according to standard National Institute of Neurological and Communicative Diseases and Stroke/Alzheimer's Disease and Related Disorders Association diagnostic criteria. ${ }^{20}$

Because we wanted to recruit patients with early $A D$ who were largely independent for activities of daily living and capable of giving informed consent, we decided to include subjects with a Clinical Dementia Rating of only $0.5-1$. Patients with MCI were diagnosed per the Modified Petersen Criteria with a Clinical Dementia Rating of $\leq 0.5$ and an ACE-M score between 78 and 88 with evidence of impairment in $\geq 1$ cognitive domain, defined as test performance on at least 2 tests (which also included the ACE-M subcomponent scores) for a given domain falling below a mean $-1.5 \mathrm{SD}$ of the normative scores for the corresponding age and education status. ${ }^{21}$ Exclusion criteria included the presence of a major neurologic disorder (stroke), medical comorbidities (cardiovascular, renal), and psychiatric illness (clinically significant anxiety and depression [Hospital Anxiety Depression 
Scale score should be $<7$ ], psychosis), similar to those in our previous study. ${ }^{19}$

\section{Imaging Protocol}

A 3T scanner (Discovery MR750w, GE Healthcare, Milwaukee, Wisconsin) with a 32-channel phased array head coil was used to acquire structural and ASL images. Structural images were obtained using a high-resolution 3D brain volume imaging sequence (3D-BRAVO) with the following parameters: TR/TE $=$ $7 / 2.98 \mathrm{~ms}$, section thickness $=1 \mathrm{~mm}$, flip angle $=12^{\circ}$, matrix size $=256 \times 256$, and voxel size $=1 \times 1 \times 1 \mathrm{~mm}^{3}$. A 3D fast spinecho pseudocontinuous ASL sequence was performed with the following acquisition parameters: TR/TE $=4852 / 10.70 \mathrm{~ms}$, flip angle $=111^{\circ}$, voxel size $=1.875 \times 1.875 \times 4$, section thickness $=4 \mathrm{~mm}, \mathrm{NEX}=3$, and postlabel delay $=2025 \mathrm{~ms}$.

\section{Data Analysis}

Structural and ASL data were processed using an off-line PC workstation. For ASL processing, quantitative CBF maps with values measured in units of milliliters $/ 100 \mathrm{~g} / \mathrm{min}$ in each subject were used. All the structural images and CBF maps were oriented in the anterior/posterior commissure line using Statistical Parameter Mapping software (SPM12; http://www.fil.ion.ucl.ac. $\mathrm{uk} / \mathrm{spm} /$ download/spm12/). CBF maps of all the participants were then normalized to a standard stereotactic space and spatially smoothed with an $8-\mathrm{mm}$ isotropic Gaussian kernel to improve the signal-to-noise ratio. Second-level statistical procedures implemented in SPM12 were used to analyze the CBF maps. The value of total intracranial volume was used as a covariate in the statistical design to correct variability among groups. Information on regional perfusion values was extracted by means of an ROI analysis. Anatomic ROIs for the posterior

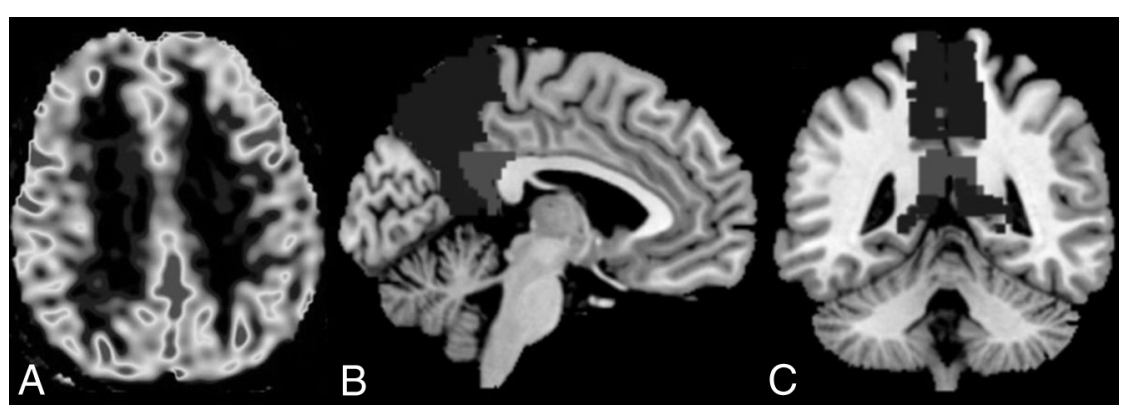

FIG 2. A, Axial ASL CBF gray-scale map. $B$ and $C$, Sagittal and coronal representative images, respectively, of the template for automated segmentation and extraction of posterior cingulate (light gray) and precuneus (dark gray) ASL CBF perfusion maps. cingulate and precuneus were defined by means of the WFU PickAtlas tool (https://www.nitrc.org/projects/wfu_pickatlas/) (Fig 2). Regional CBF was estimated using parameter extraction with MarsBaR (https://www.nitrc.org/projects/marsbar/). Values within ROIs of both sides were then averaged and tabulated for $\mathrm{HC}, \mathrm{MCI}$, and AD groups.

ERICA scoring was performed on coronally reformatted 3D-T1 BRAVO images in all subjects by 2 independent neuroradiologists (B.T. and S.K.) with 19 and 10 years of experience, respectively, at the level of the mamillary bodies as described by Enkirch et al. ${ }^{17}$ The interobserver agreement was calculated using Cohen $\kappa$ statistics. Values on the right and left sides were tabulated, and the highest ERICA score in consensus in each subject was used for further analysis.

\section{Statistical Analysis}

The $\chi^{2}$ test was used for comparison of sex differences and vascular risk factors among groups (Table 1). The clinical differentiation scores are also summarized in the same table. For comparison of age, 1-way ANOVA was performed. The ERICA score and $\mathrm{CBF}$ measures in the ROI were used to derive the predicted probability of MCI and AD based on a binary logistic regression model. The derived measures were used for generating the receiver operating characteristic curves, and the area under the curve was used to compare them (Table 2 and Fig 3). Statistical analyses were performed using the software SPSS for Windows, Version 21.0 (IBM, Armonk, New York).

\section{RESULTS}

The demographics, clinical cognitive scores, and vascular risk factors of each group are summarized in Table 1. There was no significant difference in age, sex, or vascular risk factors among these 3 cohorts.

The average highest ERICA score was $0.71 \pm 0.46$ in $\mathrm{HC}, 0.90 \pm 0.64$ in MCI, and $2.15 \pm 0.83$ in AD. There was overall substantial interrater agreement for ERICA scoring (Cohen $\kappa=0.783$ for the right side, 0.777 for the left side, and 0.797 for the highest ERICA score). The mean CBF in PCG $+\mathrm{PC}$ was $46.64 \pm 8.4,39.08 \pm 6.6$, and $36.51 \pm 8.4 \mathrm{~mL} / 100 / \mathrm{min}$ in $\mathrm{HC}$, $\mathrm{MCI}$, and $\mathrm{AD}$, respectively (Table 2 ).

Table 1: Demographic data, comorbidities, and clinical scores of subjects

\begin{tabular}{|c|c|c|c|c|c|c|}
\hline \multirow[b]{2}{*}{ Characteristic } & \multirow[b]{2}{*}{$\mathrm{HC}(n=21)$} & \multirow[b]{2}{*}{$\mathrm{MCl}(n=20)$} & \multirow[b]{2}{*}{$\mathrm{AD}(n=19)$} & \multicolumn{3}{|c|}{$P$ Value } \\
\hline & & & & $\mathrm{MCl}$ versus $\mathrm{HC}$ & $A D$ versus $H C$ & $A D$ versus $M C l$ \\
\hline Sex (male/female) & $11: 10$ & $11: 9$ & $11: 8$ & .87 & .77 & .88 \\
\hline Age (mean $\pm S D)(y r)$ & $64.57 \pm 5.74$ & $66.75 \pm 4.08$ & $66.68 \pm 5.31$ & .37 & .66 & 1.00 \\
\hline H/o hypertension & $5 / 21$ & $7 / 20$ & 9/19 & .33 & .11 & .32 \\
\hline H/o diabetes mellitus & $5 / 21$ & $8 / 20$ & $10 / 19$ & .22 & .06 & .32 \\
\hline $\begin{array}{l}\text { RAVLT cumulative learning score } \\
\text { (mean) }\end{array}$ & $48.52 \pm 8.46$ & $33.47 \pm 10.07$ & $25.21 \pm 7.15$ & $<.001$ & $<.001$ & .035 \\
\hline RAVLT 20-min recall score (mean) & $10.00 \pm 2.82$ & $5.41 \pm 3.74$ & $1.36 \pm 1.73$ & $<.001$ & $<.001$ & .001 \\
\hline ACE-M & $93.38 \pm 4.11$ & $81.12 \pm 11.09$ & $72.00 \pm 11.09$ & $<.001$ & $<.001$ & .020 \\
\hline
\end{tabular}

Note:-H/O indicates History of; RAVLT, Rey auditory verbal learning test; ACE-M, Addenbrooke's Cognitive examination. Significant $P$ values are highlighted in bold. 


\begin{tabular}{lcccccc}
\hline & & & & \multicolumn{2}{c}{$\begin{array}{c}\text { Predicted Probability of Diagnosis (Area under } \\
\text { the Curve of ROC) with } P \text { Values in Parentheses }\end{array}$} \\
\cline { 3 - 7 } \multicolumn{1}{c}{ Parameters } & HC (Mean) & MCI (Mean) & AD (Mean) & MCI vs HC & AD vs HC & AD vs MCI \\
\hline ERICA (0-3) & $0.71 \pm 0.46$ & $0.90 \pm 0.64$ & $2.15 \pm 0.83$ & $0.571(.493)$ & $0.887(<.001)$ & $0.838(<.001)$ \\
CBF in PCG + PC (mL/100 g/min) & $46.64 \pm 8.4$ & $39.08 \pm 6.6$ & $36.51 \pm 8.4$ & $0.776(.002)$ & $0.810(<.001)$ & $0.589(.339)$ \\
Combined ERICA and CBF & & & & $0.781(.002)$ & $0.957(<.001)$ & $0.829(<.001)$ \\
\hline
\end{tabular}

Note:-ROC indicates receiver operating characteristic. Significant $P$ values are highlighted in bold.

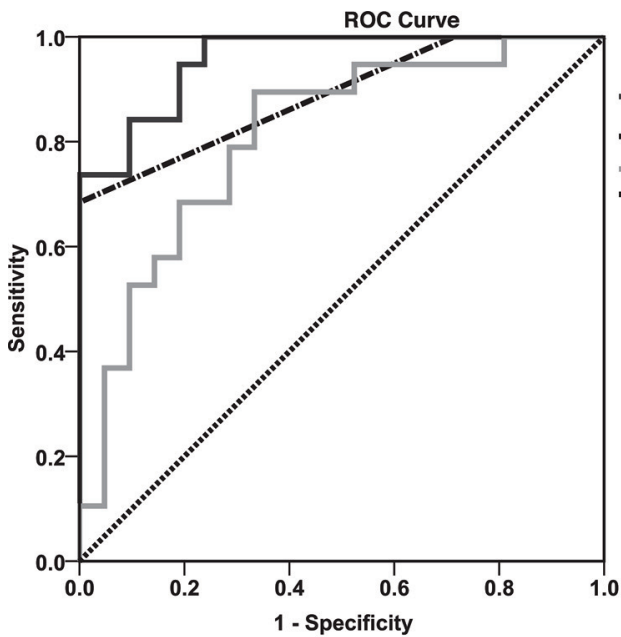

A

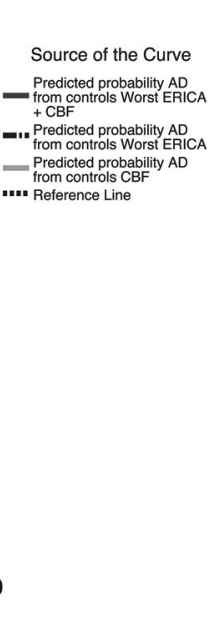

Diagonal segments are produced by ties.

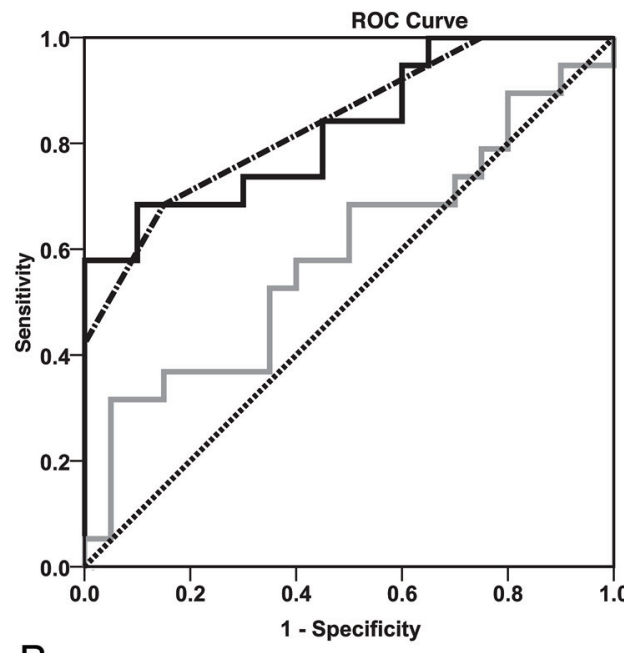

B

Diagonal segments are produced by ties.

Source of the Curve Predicted probability AD
from MCI Worst ERICA + -.. Predicted probability AD from MCI Worst ERICA
- Predicted probability $A D$ .... Reference Line

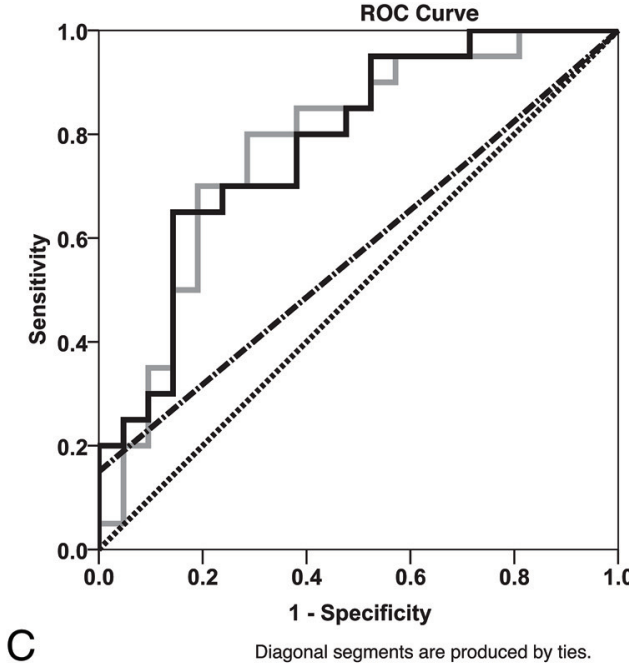

Source of the Curve Predicted probability MCI
from controls Worst ERICA -.. Predicted probability MCI Credicted probability $\mathrm{MCI}$ from controls CBF Reference Line

FIG 3. Receiver operating characteristic curves for predicted probability from ERICA (dot-dash lines) and regional CBF (light gray continuous lines) and a combination of both (black continuous lines). $A D$ versus $H C(A), A D$ versus $M C I(B), M C l$ versus $H C(C)$.

Both ERICA scores and mean CBF were highly predictive of distinguishing $\mathrm{AD}$ from $\mathrm{HC}$, with a predicted probability of 0.887 $(P<.001)$ and $0.810(P<.001)$, respectively. How-ever, combining the ERICA score and mean CBF significantly increased the discriminatory power between $\mathrm{AD}$ and $\mathrm{HC}$ with the predicted probability of $0.957(P<.001)$ (Fig $3 A)$. While the ERICA score was found to be useful in distinguishing MCI from AD (predictive probability of $0.838, P<.001$ ), regional mean $\mathrm{CBF}$ was not useful to differentiate these 2 groups (predictive probability of $0.589, P=.339)$. Marginal reduction of predictive probability $(0.829$,
$P<.001)$ was observed after a combination of ERICA scores and CBF without loss of discriminant ability (Fig $3 B$ ).

Significantly, the ERICA score was not found to be effective in distinguishing MCI from $\mathrm{HC}$ (predictive probability $=0.571$, $P=.493)$. On the contrary, regional CBF demonstrated a higher predictive probability of $0.776(P=.002)$ to differentiate these groups. Combined ERICA scores and CBF maintained this predictive power, with a marginal increase of the predictive probability of $0.781(P=.002)$. (Fig 3C).

The results are summarized in Table 2 and Fig 3. 


\section{DISCUSSION}

ASL is a noninvasive technique for cerebral perfusion measurement using electromagnetically labeled arterial water as a diffusible tracer. Several ASL perfusion studies have highlighted regional hypo- and hyperperfusion in multiple areas of the brain in MCI-AD. ${ }^{7-13}$ However, due to the variability of clinical subsets studied and the usage of nonreplicable sequences, these studies were not directly comparable. ${ }^{13}$ In the past, differences in technical implementation schemes and lack of standardized protocols hindered the routine use of ASL as a robust perfusion method in the clinical setting. International consensus guidelines have been developed recently for standardized ASL acquisitions. ${ }^{22}$ A 3D fast spin-echo pseudocontinuous ASL with a spiral readout is now generally considered the standard method of ASL in clinical research. In this study, we used a 3D fast spin-echo pseudocontinuous ASL technique on 3T MR imaging to investigate regional perfusion differences in patients with $\mathrm{MCI}$ and $\mathrm{AD}$ compared with HC. ROIs were extracted using atlas-based semiautomated techniques to avoid errors due to manual contouring.

Although several brain areas have been shown to be involved, one of the most consistent regions of hypoperfusion reported in MCI-AD is the PCG and anterior PC. ${ }^{10,11,13}$ Identification of hypoperfusion in the PCG + PC has been reported to have a sensitivity of $91 \%$ and a specificity of $80 \%$ for diagnosing AD. ${ }^{23}$ This method is in contrast to inclusion of a combination of different regions in subjects with amnestic and dysexecutive MCI, in which an accuracy of only $60 \%-70 \%$ was observed. ${ }^{24}$ From the existing literature, it is not clear whether the hypoperfusion is the cause or consequence of the disease process. ${ }^{25}$ In our study, the regional mean CBF in PCG + PC was 46.64, 39.08, and $36.51 \mathrm{~mL} / 100 / \mathrm{min}$ in $\mathrm{HC}, \mathrm{MCI}$, and $\mathrm{AD}$, respectively, and it performed well in distinguishing $\mathrm{AD}-\mathrm{HC}$ and MCI-HC, but not between MCI and $\mathrm{AD}$. This finding may be because the perfusion deficit in the PCG + PC occurs early in the course of this dementing illness spectrum, and this particular feature may be used as a reliable marker to delineate patients with $\mathrm{MCI}$ and $\mathrm{AD}$ from $\mathrm{HC}$. However, from MCI to AD transformation, the perfusion reduction in these regions may be less remarkable and hence may not have significant diagnostic utility as a stand-alone measure.

There is no consistent correlation established between CBF and gray matter changes in the corresponding regions in patients with $\mathrm{AD}$ and $\mathrm{MCI}{ }^{13}$ In the initial stages of $\mathrm{AD}$, changes such as intraneuronal alterations in the form of neurofibrillary tangles and neuropil threads are observed more frequently in the entorhinal cortex compared with hippocampus. ${ }^{26}$ This accumulation may be noted in other conditions, including normal aging, and when this is associated with neuronal loss, it leads to global cognitive decline. MR volumetric studies have also shown entorhinal cortex volume loss in dementia due to Alzheimer spectrum diseases. ${ }^{27,28}$ It has been shown in studies that in early stages of $\mathrm{AD}$, certain areas such as the prefrontal cortex or medial temporal lobe structures would eventually have the ability to compensate for the cognitive decline. ${ }^{29}$ Simultaneously, perfusion deficits might also be present from the very early preclinical phases of $\mathrm{AD}$, including $\mathrm{MCI}$ at risk of conversion, persisting into the advanced stages of the disease, demonstrating a progressive hypoperfusion with disease development, leading to brain atrophy and underlying neuronal loss that correlates with cognitive and functional decline. ${ }^{13}$ With regard to the pathophysiologic interpretation of the regional hypoperfusion consistently found in the PCG + PC, in comparison with ERICA, controversy exists about whether this is a cause or consequence of the disease process. ${ }^{25}$ It is well known that risk factors such as ischemic stroke, amyloid angiopathy, atherosclerosis, hypertension, diabetes, and cardiac disease are repeatedly implicated in the risk of MCI converting to AD. ${ }^{30}$ While $\mathrm{AD}$ and vascular dementia have traditionally been considered as independent entities, there is growing evidence implicating vascular pathology in $\mathrm{AD}$ to the extent of suggesting an overlap between $\mathrm{AD}$ and vascular dementia. ${ }^{31}$ Moreover, evidence from aging and stroke studies suggests that chronic brain hypoperfusion is the key pathophysiology that leads to neurodegeneration and consequent cognitive decline. ${ }^{25}$ Neuropsychologic correlations have also been established between perfusion measures in the parieto-occipital region and the parietal cortex along with the PCG + PC. ${ }^{32}$ Hypoperfusion may lead to changes in cortical thickness, as measured on structural MR imaging scans, in areas most vulnerable to aging (medial prefrontal and pericentral cortices) as well as in areas associated with amyloid aggregation (eg, occipitotemporal and basal temporal cortices), more so in apolipoprotein E4 carriers. ${ }^{33}$ Hence, there is the necessity to address disparate or nonconcordant topographic areas rather than looking at concordant brain volumes underlying areas of hypoperfusion while attempting to combine hypoperfusion and volumetric measures as with ERICA.

Compared with conventional MR imaging-medial temporal lobe atrophy scoring, which reported a sensitivity of $81 \%$ and a specificity of $67 \%$, ERICA had a higher sensitivity of $83 \%$ and specificity of $98 \%$ in differentiating AD. ${ }^{17,34}$ ERICA is also easier to comprehend and score compared with the more complex medial temporal lobe atrophy score. It is also reliable and reproducible as confirmed in our study. The tentorial cleft sign (Fig $1 C$ ), which indicated an ERICA score of $\geq 2$, had a high diagnostic accuracy for AD. ${ }^{17}$ In our study, the average ERICA score of patients with $\mathrm{AD}$ was 2.15. ERICA performed well in differentiating $\mathrm{AD}$ from $\mathrm{MCI}$ and $\mathrm{AD}$ from $\mathrm{HC}$, but not $\mathrm{MCI}$ from $\mathrm{HC}$. This performance may mean that significant parahippocampal atrophy does not happen early in MCI and probably is a much later occurrence compared with CBF reduction in PCG + PC in the course of progression of dementia. This hypothesis is further validated by the observations of neuropathology studies by Braak and Braak published in $1991 .^{26}$

ASL hypoperfusion abnormality in PCG + PC and parahippocampal atrophy may be considered as complementary imaging biomarkers to distinguish $\mathrm{MCI}$ and $\mathrm{AD}$. In our study combining the ERICA score with regional CBF in PCG + PC showed a promising trend to differentiate $\mathrm{MCI}, \mathrm{AD}$, and $\mathrm{HC}$ groups rather than individual parameters. However, MCI-AD is a more heterogeneous disease process, and not all patients with $\mathrm{MCI}$ are converters to $\mathrm{AD} .{ }^{17}$ Further prospective studies are needed to validate the usefulness of combined ERICA and regional CBF in predicting this conversion. Although the pattern of results in MCI-HC comparison indicates better utility of CBF PCG + PC as opposed to the ERICA score, nosologically, there are important considerations while stratifying $\mathrm{MCI}$ at risk of $\mathrm{AD}$ using 
biomarkers. Per available criteria, to identify MCI at an intermediate or higher likelihood of $\mathrm{AD}$ requires combining biomarkers of - $\beta$ amyloid accumulation (PET or CSF) with markers of neuronal injury ( $\tau$, FDG-PET, structural MR imaging). ${ }^{35} \mathrm{~A}$ combined instrument using $2 \mathrm{MR}$ imaging markers of neuronal injury rather than $-\beta$ amyloid accumulation precludes prediction of the proportion of the MCI cohort who are likely to convert in our series.

One also needs to consider that MCI is a heterogeneous condition and potentially could be due to other causes like vascular cognitive impairment. There is evidence in the literature available from MCI negative for $\beta$ amyloid, where common etiologies include vascular diseases and depression. ${ }^{36}$ It is possible that a subset of our MCI cohort may, with time, evolve as vascular cognitive impairment or other alternative trajectories or may potentially remain stable without converting to $\mathrm{AD}$. This possibility is perhaps part of the reason that the ERICA score did not show a significant difference between $\mathrm{MCI}$ and $\mathrm{HC}$ as opposed to the other 2 comparisons. Lack of longitudinal data to ascertain the trajectory of the MCI group with time precludes confirmation of the same in the present study and warrants future prospective follow-up studies. A relatively small sample size in our study also might have resulted in a lower ability to detect differences between $\mathrm{MCI}$ and $\mathrm{AD}$ using $\mathrm{CBF}$ alone at baseline. All these facts point to the relevance of including multiple MR imaging markers as implemented in one of our previous studies on multimodality neuroimaging. ${ }^{37}$

The study was performed on a prospectively acquired dataset with measurement of only 2 parameters, which could be easily reproduced in a clinical setting. We preferred to use computed segmentation of the ROI CBF to avoid any bias due to manual drawing of contours. However, this study had several limitations. Though ASL is a robust technique to measure cerebral perfusion, technical parameters and patient-specific factors could variably influence the CBF calculation and confound the results. Specific to dementia, one of the major confounders is the arterial transit time, which can be different for each subject due to associated vascular or nonvascular comorbidities. The possible vascular risk contributors like diabetes mellitus and hypertension were not considerably different in each subgroup in the present study (Table 1). Again, transit delay can be higher in some of the ROIs in dementia imaging like the posterior parietal lobes, leading to erroneous CBF measurements. ${ }^{38}$ The appropriately increased postlabeling delay time could be used to reduce this error. Alternatively, a multidelay ASL can potentially solve this problem, but it is not ready for prime time imaging yet. Also, a small sample size and cross-sectional study design did not permit analyzing a causal relationship between the biomarkers and the development of $\mathrm{MCI}$ and $\mathrm{AD}$, and further large longitudinal studies are needed to confirm its role in predicting progression of the disease. Additionally, head-to-head comparisons between Pittsburgh compound PET imaging and CSF biomarkers (which are currently not available at our center) and MR imaging markers would have enabled us to ascertain the sensitivity and specificity; however, this comparison remains a concern across many centers in the developing world, and only multicentric collaborative ventures can transcend the issue.

\section{CONCLUSIONS}

This study explored the utility of measuring structural and perfusion changes in specific and nonoverlapping ROIs in differentiating $\mathrm{MCI}$ and $\mathrm{AD}$. Combining regional perfusion measurements in the PCG + PC using ASL along with the ERICA score on structural imaging has the potential to be a better imaging biomarker to distinguish among Alzheimer spectrum disorders. In centers that do not have access to nuclear imaging scans including FDG Pittsburgh compound PET, this study has shown the utility of objective multimodal imaging scores to distinguish MCI at risk of progression to $\mathrm{AD}$. This use of scores has the potential to serve as a noninvasive tool to stratify MCI etiologically, which is a heterogeneous entity in itself. Future studies are needed to explore the longitudinal risk of cognitive decline in MCI due to $\mathrm{AD}$ (using markers of amyloid accumulation) as opposed to MCI due to non-AD pathology, including vascular cognitive impairment, in direct correlation with ERICA and regional CBF measures. This categorization should also account for confounding factors such as age, education status, hypertension, coronary artery disease, body mass index, dyslipidemia, and impaired glucose tolerance in these groups.

Disclosures: Bejoy Thomas-RELATED: Grant: Department of Science and Technology (DST), Government of India*; Support for Travel to Meetings for the Study or Other Purposes: DST, Government of India*; UNRELATED: Grants/ Grants Pending: GE Healthcare, research grant*; Patents (Planned, Pending or Issued): applied for Indian patent*; Stock/Stock Options: Minority stocks in Wipro India. Ramshekhar N. Menon—RELATED: Grant: Department of Science and Technology, Comments: grant numbers mentioned in the article*; UNRELATED: Grants/Grants Pending: Department of Science and Technology, Comments: Details are provided in terms of grant numbers in the article.* *Money paid to institution.

\section{REFERENCES}

1. Crous-Bou M, Minguillón C, Gramunt N, et al. Alzheimer's disease prevention: from risk factors to early intervention. Alzheimers Res Ther 2017;9:71 CrossRef Medline

2. Tolppanen A-M, Taipale H, Purmonen T, et al. Hospital admissions, outpatient visits and healthcare costs of community-dwellers with Alzheimer's disease. Alzheimers Dement 2015;11:955-63 CrossRef Medline

3. Petersen RC. Mild cognitive impairment as a diagnostic entity. $J$ Intern Med 2004;256:183-94 CrossRef Medline

4. Apostolova LG. Alzheimer disease. Continuum (Minneap Minn) 2016;22:419-34 CrossRef Medline

5. Sheelakumari R, Kesavadas C, Lekha VS, et al. Menon RN structural correlates of mild cognitive impairment: a clinicovolumetric study. Neurol India 2018;66:370-36 CrossRef Medline

6. Hays CC, Zlatar ZZ, Wierenga C. The utility of cerebral blood flow as a biomarker of preclinical Alzherimer's disease. Cell $\mathrm{Mol}$ Neurobiol 2016;36:167-79 CrossRef Medline

7. Sandson TA, O'Connor M, Sperling RA, et al. Noninvasive perfusion MRI in Alzheimer's disease: a preliminary report. Neurology 1996; 47:1339-42 CrossRef Medline

8. Alsop DC, Detre JA, Grossman M. Assessment of cerebral blood flow in Alzheimer's disease by spin-labeled magnetic resonance imaging. Ann Neurol 2000;47:93-100 CrossRef Medline

9. Johnson NA, Jahng G-H, Weiner MW, et al. Pattern of cerebral hypoperfusion in Alzheimer disease and mild cognitive impairment measured with arterial spin-labeling MR imaging: initial experience. Radiology 2005;234:851-59 CrossRef Medline

10. Dai W, Lopez OL, Carmichael OT, et al. Mild cognitive impairment and Alzheimer disease: patterns of altered cerebral blood flow at MR imaging. Radiology 2009;250:856-66 CrossRef Medline 
11. Binnewijzend MAA, Kuijer JPA, Benedictus MR, et al. Cerebral blood flow measured with $3 \mathrm{D}$ pseudocontinuous arterial spinlabeling MR imaging in Alzheimer disease and mild cognitive impairment: a marker for disease severity. Radiology 2013;267:22130 CrossRef Medline

12. Bron EE, Steketee RME, Houston GC, et al. Diagnostic classification of arterial spin labeling and structural MRI in presenile early stage dementia. Hum Brain Mapp 2014;35:4916-31 CrossRef Medline

13. Sierra-Marcos A. Regional cerebral blood flow in mild cognitive impairment and Alzheimer's disease measured with arterial spin labeling magnetic resonance imaging. Int J Alzheimer's Dis 2017; 2017:5479597 CrossRef Medline

14. Verclytte S, Lopes R, Delmaire C, et al. Optimization of brain perfusion image quality by cortical surface-based projection of arterial spin labeling maps in early-onset Alzheimer's disease patients. Eur Radiol 2015;25:2479-84 CrossRef Medline

15. Tosun D, Schuff N, Jagust W, et al; Alzheimer's Disease Neuroimaging Initiative. Discriminative power of arterial spin labeling magnetic resonance imaging and $18 \mathrm{~F}$-fluorodeoxyglucose positron emission tomography changes for amyloid-beta-positive subjects in the Alzheimer's disease continuum. Neurodegener Dis 2016; 16:87-94 CrossRef Medline

16. Kaneta $\mathrm{T}$, Katsuse $\mathrm{O}$, Hirano $\mathrm{T}$, et al. Voxel-wise correlations between cognition and cerebral blood flow using arterial spin-labeled perfusion MRI in patients with Alzheimer's disease: a crosssectional study. BMC Neurol 2017;17:91 CrossRef Medline

17. Enkirch SJ, Traschütz A, Müller A, et al. The ERICA score: an MR imaging-based visual scoring system for the assessment of entorhinal cortex atrophy in Alzheimer disease. Radiology 2018;288:226-33 CrossRef Medline

18. Mathuranath PS, Hodges JR, Mathew R, et al. Adaptation of the ACE for a Malayalam speaking population in southern India. Int J Geriat Psychiatry 2004;19:1188-94 CrossRef Medline

19. Menon R, Lekha V, Justus S, et al. A pilot study on utility of Malayalam version of Addenbrooke's Cognitive Examination in detection of amnestic mild cognitive impairment: a critical insight into utility of learning and recall measures. Ann Indian Acad Neurol 2014;17:420 CrossRef Medline

20. McKhann G, Drachman D, Folstein M, et al. Clinical diagnosis of Alzheimer's disease: report of the NINCDS-ADRDA Work Group under the auspices of Department of Health and Human Services Task Force on Alzheimer's disease. Neurology 1984; 34:939-44 CrossRef Medline

21. Petersen RC, Stevens JC, Ganguli M, et al. Practice parameter: early detection of dementia: mild cognitive impairment (an evidencebased review) - report of the Quality Standards Subcommittee of the American Academy of Neurology. Neurology 2001;56:1133-42 CrossRef Medline

22. Alsop DC, Detre JA, Golay X, et al. Recommended implementation of arterial spin-labeled perfusion MRI for clinical applications: a consensus of the ISMRM Perfusion Study group and the European Consortium for ASL in Dementia. Magn Reson Med 2015;73:10216 CrossRef Medline
23. Yoshiura $\mathrm{T}$, Hiwatashi A, Noguchi $\mathrm{T}$, et al. Arterial spin labelling at 3-T MR imaging for detection of individuals with Alzheimer's disease. Eur Radiol 2009;19:2819-25 CrossRef Medline

24. Chao LL, Pa J, Duarte A, et al. Patterns of cerebral hypoperfusion in amnestic and dysexecutive MCI. Alzheimer Dis Assoc Disord 2009;23:245-52 CrossRef Medline

25. Austin BP, Nair VA, Meier TB, et al. Effects of hypoperfusion in Alzheimer's disease. J Alzheimer's Dis 2011;26(Suppl 3):123-33 CrossRef Medline

26. Braak H, Braak E. Neuropathological stageing of Alzheimer-related changes. Acta Neuropathol 1991;82:239-59 CrossRef

27. Jessen F, Feyen L, Freymann K, et al. Volume reduction of the entorhinal cortex in subjective memory impairment. Neurobiol Aging 2006;27:1751-56 CrossRef Medline

28. deToledo-Morrell L, Stoub TR, Bulgakova M, et al. MRI-derived entorhinal volume is a good predictor of conversion from MCI to AD. Neurobiol Aging 2004;25:1197-1203 CrossRef Medline

29. Chen W, Song $X$, Beyea $S$, et al. Advances in perfusion magnetic resonance imaging in Alzheimer's disease. Alzheimers Dement 2011; 7:185-96 CrossRef Medline

30. Mazza M, Marano G, Traversi G, et al. Primary cerebral blood flow deficiency and Alzheimer's disease: shadows and lights. $J$ Alzheimers Dis 2011;23:375-89 CrossRef Medline

31. Zhang Q, Stafford RB, Wang Z, et al. Microvascular perfusion based on arterial spin labeled perfusion MRI as a measure of vascular risk in Alzheimer's disease. J Alzheimers Dis 2012;32:677-87 CrossRef

32. Alsop DC, Detre JA, Grossman M. Assessment of cerebral blood flow in Alzheimer's disease by spin-labeled magnetic resonance imaging. Ann Neurol 2000;47:93-100 CrossRef

33. Espeseth T, Westlye LT, Fjell AM, et al. Accelerated age-related cortical thinning in healthy carriers of apolipoprotein $\mathrm{E}$ epsilon 4 . Neurobiol Aging 2008;29:329-40 CrossRef Medline

34. Scheltens P, Leys D, Barkhof F, et al. Atrophy of medial temporal lobes on MRI in “probable” Alzheimer's disease and normal ageing: diagnostic value and neuropsychological correlates. J Neurol Neurosurg Psychiatry 1992;55:967-72 CrossRef Medline

35. Albert MS, DeKosky ST, Dickson D, et al. The diagnosis of mild cognitive impairment due to Alzheimer's disease: recommendations from the National Institute on Aging-Alzheimer's Association workgroups on diagnostic guidelines for Alzheimer's disease. Alzheimers Dement 2011;7:270-79 CrossRef Medline

36. Landau SM, Horng A, Fero A, et al. Alzheimer's Disease Neuroimaging Initiative. Amyloid negativity in patients with clinically diagnosed Alzheimer disease and MCI. Neurology 2016;86:1377-85 CrossRef Medline

37. Sheelakumari R, Sarma SP, Kesavadas C, et al. Multimodality neuroimaging in mild cognitive impairment: a cross-sectional comparison study. Ann Indian Acad Neurol 2018;21:133-39 CrossRef Medline

38. Alsop DC, Dai W, Detre JA, et al. Arterial spin labeling blood flow MRI: its role in the early characterization of Alzheimer's disease. $J$ Alzheimers Dis 2010;20:871-80 CrossRef Medline 